\title{
The effect of trabeculectomy surgery on the central visual field in patients with glaucoma using microperimetry and optical coherence tomography
}

\author{
Gokulan Ratnarajan $^{1,3} \cdot$ Jasleen K. Jolly $\mathbb{1 0}^{1,2} \cdot$ Imran H. Yusuf $^{1,2} \cdot$ John F. Salmon ${ }^{1,2}$ \\ Received: 14 November 2017 / Revised: 1 March 2018 / Accepted: 2 March 2018 / Published online: 30 April 2018 \\ (c) The Royal College of Ophthalmologists 2018
}

\begin{abstract}
Purpose To determine the functional and structural effects of trabeculectomy surgery on patients with advanced glaucoma and central visual field defects in the early post-operative period.

Methods Thirty consecutive adult subjects with advanced glaucoma requiring trabeculectomy surgery and an established visual field defect within $10^{\circ}$ of fixation underwent microperimetry (MAIA MP-1, CenterVue, Padova, Italy) and optic disc optical coherence tomography (OCT) imaging (Spectralis, Heidelberg Engineering, Germany) pre-operatively, and 1 month and 3 months following trabeculectomy surgery. Main outcome measures were post-trabeculectomy change in mean threshold on microperimetry and nerve fibre layer thickness on OCT. Fellow eyes were used as controls.

Results The mean change in MP average threshold values from pre-operative to post-operative was $0.6 \pm 1.9 \mathrm{~dB}$ for treated eyes and $0.1 \pm 1.3 \mathrm{~dB}$ for control eyes $(p=0.14)$ at 1 month and $0.2 \pm 2.3$ and $-0.3 \pm 1.6 \mathrm{~dB}$ at 3 months $(p=0.22)$. Mean change in global nerve fibre layer thickness was -0.6 and $-0.5 \mu \mathrm{m}$ for operated and control eyes, respectively $(p=0.83)$, at 1 month and 0.8 and $-0.4 \mu \mathrm{m}$ at 3 months $(p=0.88)$. The kappa agreement for structure-function correlation between OCT and MP was 0.735 (confidence interval 0.59-0.88) $(p<0.005)$.

Conclusions Central visual function and retinal nerve fibre layer thickness appear to be preserved in glaucoma patients with central visual field defects undergoing trabeculectomy surgery in the early post-operative period. These data may inform glaucoma surgeons considering trabeculectomy surgery in this patient group.
\end{abstract}

\section{Introduction}

Glaucoma is the leading cause of irreversible blindness worldwide [1]. Intraocular pressure (IOP) is the most influential modifiable risk factor in glaucoma. Raised IOP has been demonstrated to increase the risk of glaucoma in

Electronic supplementary material The online version of this article (https://doi.org/10.1038/s41433-018-0095-2) contains supplementary material, which is available to authorized users.

Gokulan Ratnarajan

g.ratnarajan@gmail.com

1 Oxford Eye Hospital, Oxford University Hospitals NHS Trust and NIHR Biomedical Research Centre, Oxford, UK

2 Nuffield Laboratory of Ophthalmology, University of Oxford, Oxford, UK

3 Present address: Corneo-plastic unit, Queen Victoria Hospital NHS Foundation Trust East Grinstead UK patients with ocular hypertension [2], and visual loss in patients with glaucoma [2-4]. Reducing the IOP in glaucoma has been proven to reduce the risk of visual field loss. Strategies to reduce IOP include topical drops, laser or surgery [3, 4].

Trabeculectomy is a surgical intervention proven to achieve long-term reduction in IOP in patients with glaucoma [5]. It is indicated in patients with a high risk of progressive visual field loss considered refractory to medical treatment. Control of IOP in patients with glaucomatous visual loss affecting the central visual field is particularly critical [6]. However, there is concern that transient or permanent central visual loss may occur as a consequence of trabeculectomy surgery in this patient group [7-12]. Outcome measures in these studies were based on standard automated perimetry indices to detect change in visual function, which may not identify subtle changes in the central visual field postoperatively. 
Microperimetry is a diagnostic technique in which a light stimulus is projected onto specific retinal locations using eye tracking technology, mapping the retinal threshold sensitivity topographically across the macula [13]. It is considered more sensitive than standard automated perimetry for the evaluation of scotomas, correlating closely with structural changes on OCT [14]. There are no studies that have specifically evaluated the macular sensitivity using microperimetry in patients with glaucoma and an established central visual field defect following trabeculectomy surgery. Spectral domain OCT has been demonstrated to reproducibly evaluate the peripapillary retinal nerve fibre layer thickness and ganglion cell-inner plexiform layer (GCIPL) in patients with glaucoma, both for diagnosis of glaucoma and detection of disease progression over time $[15,16]$.

We sought to determine the functional and structural effects of trabeculectomy surgery on the central visual field in glaucoma patients with an established visual field defect in the early post-operative period using microperimetry and optical coherence tomography imaging.

\section{Methods}

\section{Patient selection}

Thirty consecutive adult patients with advanced glaucoma affecting the central visual field and requiring trabeculectomy surgery were recruited from the glaucoma clinic in a tertiary referral centre (Oxford Eye Hospital, Oxford, UK). Fellow eyes were used as a control. All patient participants indicated their signed informed consent after explanation of the nature of the study. The study was approved by an ethics and institutional review board committee (15/NW/0231) prior to the study period. The research was conducted in accordance with the tenets of the Declaration of Helsinki.

Inclusion criteria for participation were: willing participant able to provide informed consent, male or female aged 18 years or over, open-angle glaucoma, patient selected for trabeculectomy surgery and central visual field loss on 24-2 Humphrey visual field analysis (defined as: three contiguous missed points within the central $10^{\circ}$ of fixation with a least one point $<0.01$, corrected pattern standard deviation $<0.05$, outside normal limits for Glaucoma Hemifield Test). Exclusion criteria were: inability to perform perimetry, macular pathology in affected eye (macular hole, agerelated macular degeneration, drusen, epiretinal membrane, etc.), non-glaucomatous optic nerve pathology (drusen, ischaemic optic neuropathy, etc.), history of intraocular inflammation or trauma, previous intraocular surgery excluding cataract surgery and secondary glaucomas
Table 1 Description of baseline characteristics of patients

\begin{tabular}{lll}
\hline & Mean & Range \\
\hline Age (years) & 69 & $24-85$ \\
Visual acuity (LogMAR) & 0.14 & -0.2 to 0.8 \\
Intraocular pressure (mm Hg) & 22.2 & $10-42$ \\
HVF mean deviation (dB) & -12.62 & -2.37 to -23.79 \\
Topical medications & 2.76 & $1-4$ \\
Sex & 16 Male, 12 Female \\
Laterality & 11 Right, 17 Left \\
Mitomycin-C & 10 eyes \\
\hline
\end{tabular}

LogMAR logarithm of the minimum angle of resolution, $H V F$ Humphrey visual field

(traumatic, pseudoexfoliation, neovascular, pigmentary, etc.). Exclusion criteria for the fellow eye was an inability to perform perimetry.

Table 1 presents a description of baseline characteristics of patients, including mean visual acuity, pre-operative intraocular pressures, pre-operative mean deviation of Humphrey automated visual field analysis, number of topical medications at baseline, age, sex and use of mitomycin-C.

\section{Study protocol}

All patients underwent baseline assessment at the time of selection for trabeculectomy surgery including visual acuity measurement, with slit lamp biomicrosopy and Goldmann applanation tonometry. Dilated examination following instillation of tropicamide $1 \%$ eye drops was undertaken on all patients followed by lens/cataract assessment using the lens opacification classification criteria II and stereoscopic optic disc examination. The Heidelberg Spectralis OCT machine was used. The optic disc was scanned using peripapillary retinal fibre layer thickness (RNFL) using a circle of $12^{\circ}$. Macula scans were $30 \times 15^{\circ}$ with 37B scans with a distance of $120 \mu \mathrm{m}$ between the cross-sections.

\section{Microperimetry examination protocol}

Visual field analysis was conducted with MAIA microperimetry (Centervue SpA, Padova, Italy) following $20 \mathrm{~min}$ adaptation in mesopic conditions (light levels below $1 \mathrm{~lx}$ ). All field plots had a reliability score of $85 \%$ or greater. The MAIA performs fundus tracking using a line-scanning laser ophthalmoscope (SLO) with a super-luminescent diode illumination that has a central wavelength of $850 \mathrm{~nm}$. This facilitated registration so the same points were retested at follow-up examinations. The MAIA uses a Goldmann size III target presented against a background of $1.27 \mathrm{~cd} / \mathrm{m}^{2}$ 


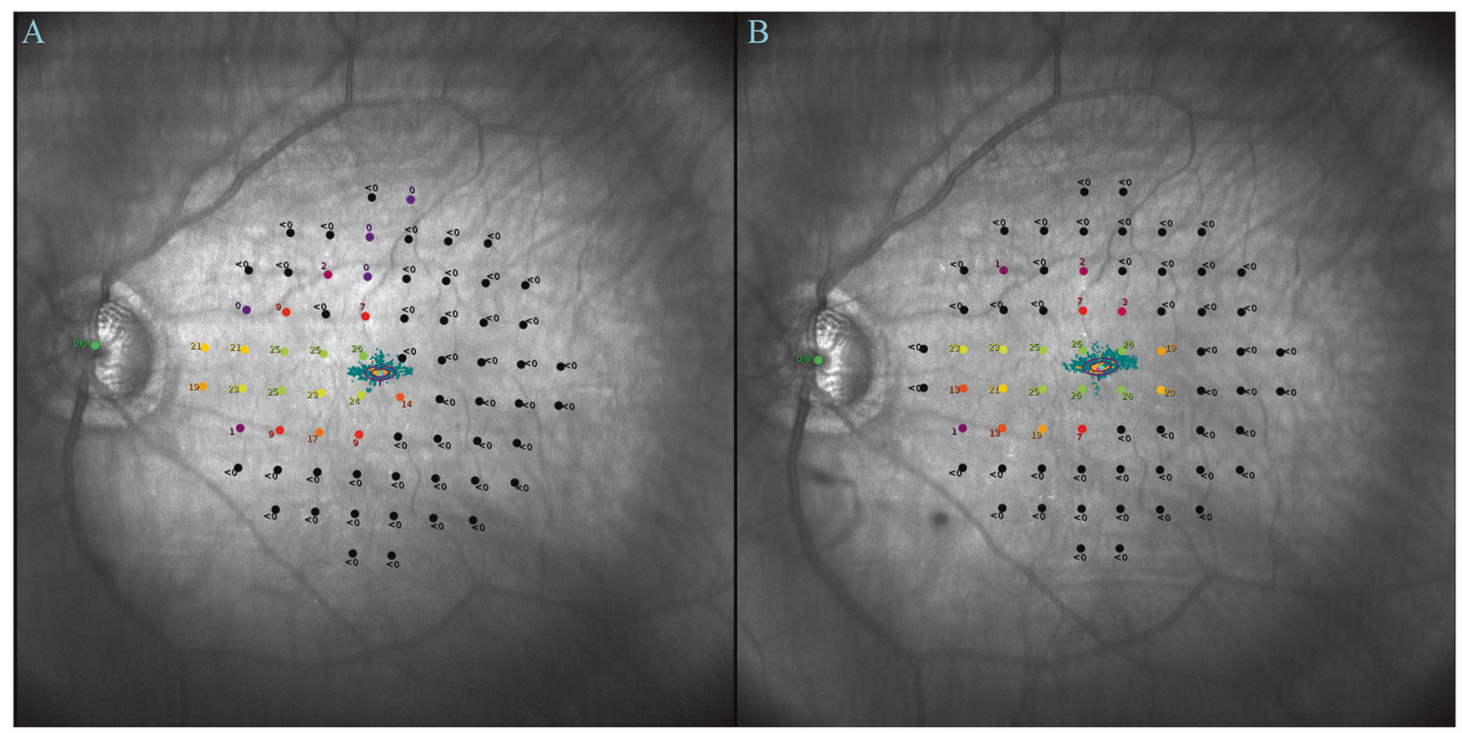

C

$8^{8} \quad 8^{7}$

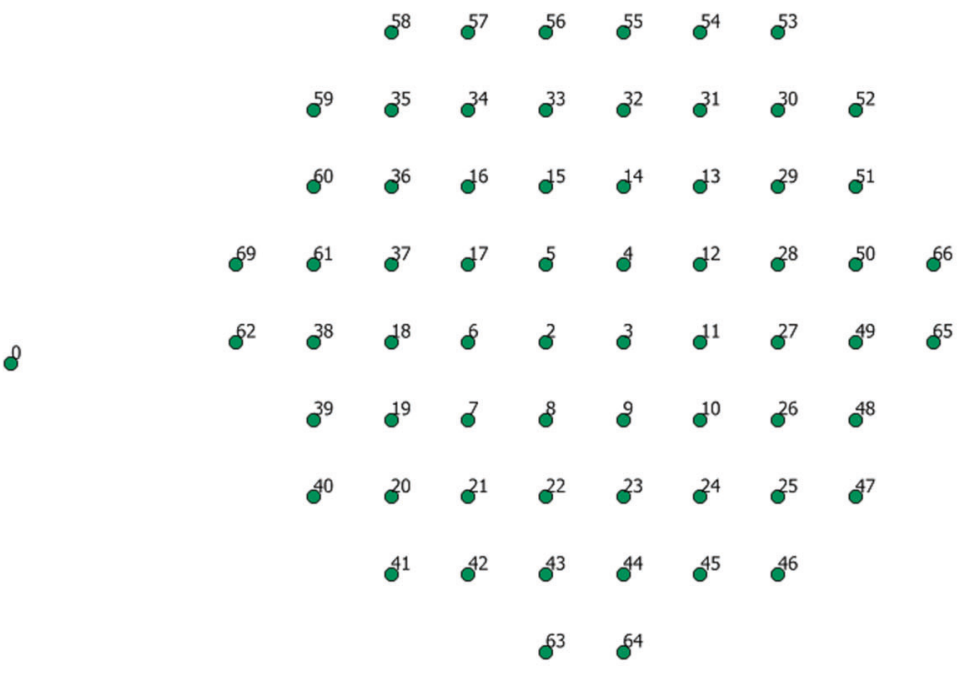

Fig. 1 10-2 MAIA plot superimposed on the SLO fundus image showing threshold at each point tested and spread of fixation points throughout testing (blue dots). a Baseline visit, b 3 months following surgery and c corresponding point IDs for each location

using a 4-2 threshold strategy. A short initial training test was conducted at baseline. This was followed by two 10-2 tests covering a similar area to a Humphrey $10^{\circ}$ visual field as shown in Fig. 1 to reduce the 'learning effect' and increase the study test accuracy. All tests were undertaken on both eyes. Each point has an assigned ID allowing point by point serial analysis.

Trabeculectomy was performed using fornix-based conjunctival flap and a $4 \mathrm{~mm}$ by $3 \mathrm{~mm}$ scleral flap closed with non-adjustable 10-0 nylon sutures. Following trabeculectomy surgery, patients received routine post-operative care. At 1 month following surgery, each patient underwent routine clinical assessment, Goldmann IOP measurement and microperimetry on both eyes. An OCT scan of the optic disc and macula was performed on each eye following dilation with tropicamide $1 \%$. Repeat assessment using the same protocol was undertaken in both eyes, in all patients, at 3 months following trabeculectomy surgery.

\section{Statistical analysis}

Statistical analysis was conducted with SPSS version 22 (IBM Corporation, New York, NY, USA). Serial analysis was conducted using a linear mixed model for microperimetry and OCT to account for missing data points. Edge effects were analysed at the edge of the scotoma for each individual patient, comparing pre-determined point ID thresholds at 3 months compared to baseline in order to 


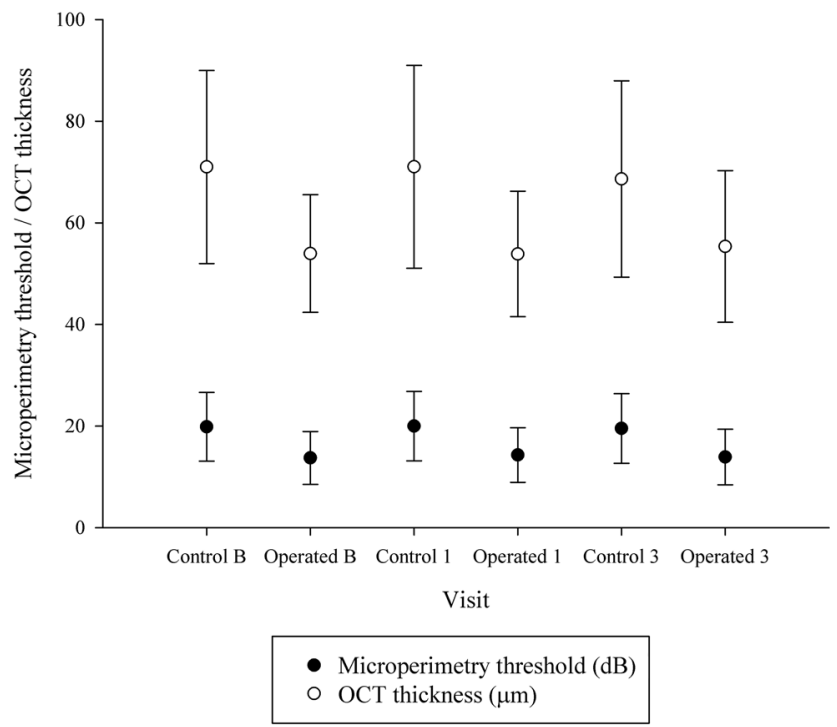

Fig. 2 A chart summarising the relation between OCT and microperimetry across all three visits in control and operated eyes. There was no significant difference in MP or OCT measurements in either the control eyes or the treated eyes across timepoints. Circles represent the mean and the error bars represent the standard deviation. $X$-axis labels: B baseline, 11 month, 33 months

assess changes at the high risk area of change (Fig. 1). High risk was defined by all points at the interface between seeing and non-seeing and was individualised for each patient.

\section{Results}

In the study eyes undergoing trabeculectomy surgery, the worse glaucoma was affecting the central visual field in all cases.

Microperimetry was completed by $n=28$ at all timepoints (two patients were unable to perform accurate tests, as shown by an accuracy score calculated during testing of $<85 \%$, for all visits and withdrawn from the study). The two baseline tests were averaged to account for patient variability and compared to tests at 1 month and 3 months. The mean pre-operative IOP in treated eyes was $22.8 \mathrm{~mm} \mathrm{Hg}$, at 1 month was $11.7 \mathrm{~mm} \mathrm{Hg}$ and at 3 months $10.5 \mathrm{~mm} \mathrm{Hg}$. There were no cases of hypotony as defined purely by IOP $(<6 \mathrm{~mm} \mathrm{Hg})$. There was no change in fellow eye IOP during this period. In addition, no significant post-operative complications were identified (such as high IOP or excessive intraocular inflammation). Mean visual acuity (LogMAR) was reduced at week 1 at 0.26 , although this improved at 1 month to 0.15 , and at 3 months was 0.07 . No individual eye experienced a drop in visual acuity that had not returned to baseline by 3 months following trabeculectomy surgery.

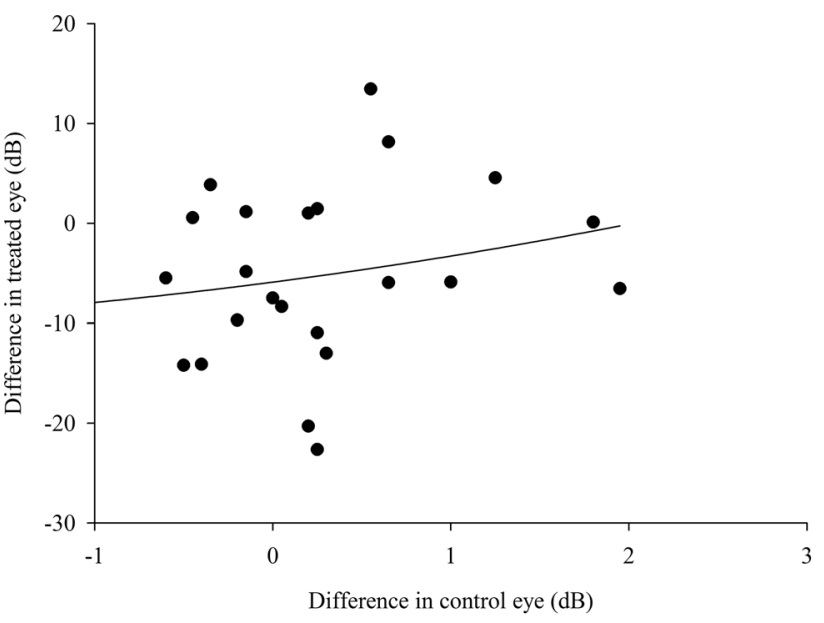

Fig. 3 Chart comparing the difference in MP mean threshold between controls and treated eyes at baseline and 3 months

The mean change in MP average threshold values from pre-operative to post-operative was $0.6 \pm 1.9 \mathrm{~dB}$ for treated eyes and $0.1 \pm 1.3 \mathrm{~dB}$ for control eyes $(p=0.14)$ at 1 month and $0.2 \pm 2.3$ and $-0.3 \pm 1.6 \mathrm{~dB}$ at 3 months, respectively $(p=0.22)$. Mean change in global nerve fibre layer thickness was -0.6 and $-0.5 \mu \mathrm{m}$ for operated and control eyes, respectively $(p=0.83)$ at 1 month and 0.8 and -0.4 $\mu \mathrm{m}$ at 3 months $(p=0.88)$. Repeated-measures analysis of variance on the mean threshold revealed that the difference between the eyes was significant as expected due to asymmetry in the severity of glaucoma between the treated and control eyes $(p=0.002)$. Change over time was not significant $(p=0.24)$ (Figs. 2 and 3 ). The coefficient of repeatability of microperimetry calculated from two tests following the learning test was $1.43 \mathrm{~dB}$. This was used to classify changes at 3 months compared to baseline as improvement, deterioration or no change (Supplementary Figure 1). This profile was similar in both eyes.

Thresholds at the edge of the scotomas followed a normal distribution (Shapiro-Wilk). Differences between baseline and 3 months were $4.57 \pm 51.12 \mathrm{~dB}(p=0.64)$ (paired $t$ test). There was no evidence of a consistent change at the edge of the scotoma.

For OCT, no difference following trabeculectomy surgery $(p=0.88)$ was found at 3 months. The difference between the eyes is significant as expected $(p=0.000)$ (Fig. 2). The mean nerve fibre layer measurements were correlated to the mean MP threshold measurements for 28 eyes at baseline (Fig. 4). The control eyes demonstrated NFL and MP values indicative of normal eyes, or with evidence of early glaucoma, and the operated eyes had more severe glaucoma. The $R$ value for control eyes was 0.664 , and 0.381 for the operated eyes. 


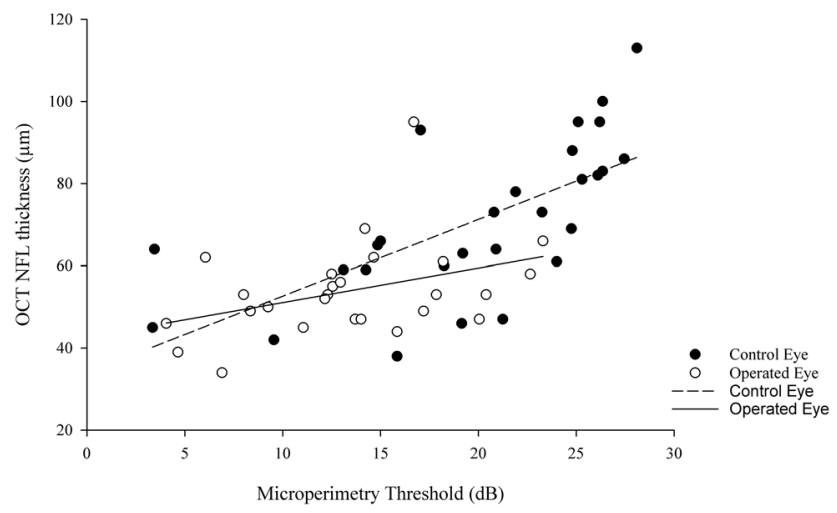

Fig. 4 Correlation between microperimetry threshold and OCT nerve fibre layer thickness for control and operated eyes

\section{Discussion}

The management of glaucoma in patients with progressive, central visual field loss refractory to medical therapy is challenging. Uncontrolled IOP in this context will result in progressive central visual field loss. Trabeculectomy surgery has been proven to achieve long-term IOP reduction in patients with glaucoma: $60 \%$ have controlled IOP with no drops, and $90 \%$ with drops at 20 years [5]. Sudden and unexplained visual loss after an intraocular procedure such as trabeculectomy surgery in patients with advanced glaucoma has been documented, and influences clinical decision making amongst ophthalmologists $[9,11]$. The risk of this phenomenon is higher in patients with 'split-fixation' visual field defects [11], particularly in those with multiple affected quadrants [9]. Consequently, many ophthalmologists defer primary trabeculectomy surgery in this patient group, although it would consider early surgical intervention if evidence supporting the stability of the central visual field were available [10]. This study may have an influence on clinical decision making in this patient group and permit more glaucoma patients with advanced central field loss to benefit from early trabeculectomy surgery [5].

Split-fixation visual field defects more often occur in patients with normal tension glaucoma in whom the starting IOP falls within the normal range $(<21 \mathrm{~mm} \mathrm{Hg})$ [17]. This patient group requires a lower target IOP following intervention to prevent progressive visual field loss from glaucoma $[18,19]$, and also typically suffer central visual field loss early in the clinical course, often by the time of presentation. These two factors in combination make trabeculectomy surgery an effective early therapeutic option in this patient group as a lower target IOP can be achieved compared to medical treatments.

This study is the first described to evaluate the central visual field of glaucoma patients with established scotomas close to fixation using microperimetry both prior to and following trabeculectomy surgery. Visual loss has been excluded in two previous series in patients with advanced glaucoma involving the central field, with standard automated perimetry in the peri-operative period $[11,20]$. A larger series with a longer follow-up period of patients undergoing trabeculectomy surgery identified a $2 \%$ risk of 'snuff out' of the central field with severe visual loss in patients undergoing trabeculectomy surgery without an identifiable cause [9]. In a series of 508 eyes, 10 eyes had post-operative central visual loss secondary to hypotony maculopathy or 'wipe out' (2\%) [21]. Pre-operative splitfixation visual field defects were a risk factor for this occurrence, increasing risk was associated with $>1$ quadrant of split-fixation defects present [9]. A further series of 117 eyes identified no cases of 'snuff out' of the central visual field following trabeculectomy surgery in patients with advanced glaucoma, although it did identify a cause of severe visual loss in $6 \%$ of cases, associated with high IOPs post-operative and surgical complications [12]. This study may be inadequately powered to detect the phenomenon of 'snuff out' of the central field in glaucoma with a suggested incidence of around $2 \%$. These reported studies utilised standard automated perimetry (SAP) and visual acuity as outcome measures which may not have identified sutble and persistent changes in central visual function possible with microperimetric assessment [14].

Microperimetry is a sensitive diagnostic tool enabling detailed and reproducible functional assessment of the central visual field [14]. It has been compared directly with SAP in patients with glaucoma and paracentral visual field defects [14]. Whilst a correlation between MP and SAP was demonstrated, MP was found to be more sensitive in detecting subtle abnormalities of visual function in areas of structural damage of the retinal nerve fibre layer on OCT imaging [14]. It was demonstrated that $21 \%$ of patients with normal SAP quadrants had an abnormal corresponding MP result. In these subjects, OCT demonstrated a structural defect in $75 \%$ of such quadrants [14]. A further study identified that MP was able to detect $100 \%$ of central scotomas corresponding to focal nerve fibre layer defects, found only in $56 \%$ of eyes using SAP [22]. Given the sensitivity of microperimetry as described in such studies, this series adds to the evidence suggesting stability of the central visual field in glaucoma patients with existing central visual field defects undergoing trabeculectomy surgery.

The influence of trabeculectomy on retinal nerve thickness has been discussed in the literature with varying conclusions. Some studies report an increase in RNFL thickness post-trabeculectomy surgery [23], although some studies have not detected an increase in thickness. [24, 25]. A series of 17 glaucoma patients undergoing trabeculectomy surgery found short-term fluctuations in RNFL thickness, increasing in the post-operative period, but returning to normal within 3 months [23]. In this study, a 
small increase in RNFL in the trabeculectomy group was detected at 3 months but this change was not statistically significant. Controversy remains as to the effect of test-retest variability on this as reflected by differences in MD that are not necessarily mirrored in pattern standard deviation [26, 27]. One study found an association between progressive RNFL thinning after trabeculectomy surgery and sustained lamina cribrosa displacement reduction [28].

The main weakness of this study is the relatively small sample size. As a consequence, no definitive conclusions can be made in respect of the phenomenon of 'snuff out' of the central visual field, where an incidence of $2-4 \%$ has been reported in these circumstances [21]. In addition, we acknowledge the relatively infrequent use of mitomycin-C in this series may conceivably reduce the incidence of postoperative hypotony, but this does reflect the clinical practice in this centre. Visual loss following trabeculectomy may be attributed in part to the effects of hypotony in the postoperative period. The risk of post-operative visual loss may therefore be higher in units which perform mitomycin-caugmented trabeculectomy surgery in a higher percentage of patients. Hypotony may only occur in $1.7 \%$ of patients in mitomycin-augmented trabeculectomy surgery [29]. The main strength of the study is the novel use of MP to assess retinal function after trabeculectomy. MP is a more sensitive measure of retinal function than SAP [14]. The stability of retinal function post trabeculectomy in the study will help guide clinicians who are considering trabeculectomy in patients with central visual field defects.

\section{Summary}

\section{What was known before:}

- Trabeculetomy surgery has been associated with a loss of central retinal sensitivity in the post-operative period in some patients with advanced glaucoma.

- The effect of trabeculectomy surgery on the retinal nerve fibre layer thickness as measured by optical coherence tomography in the post-operative period is unknown.

- Microperimetry is a novel and sensitive diagnostic technique that may be used to detect subtle changes in central retinal function.

\section{What this study adds:}

- Microperimetry did not detect any significant change in central retinal sensitivity in the early post-operative period following trabeculectomy surgery in patients with advanced glaucoma.

- Optical Coherence Tomography imaging did not detect any significant change in retinal nerve fibre layer thickness following trabeculectomy surgery.
- This study may inform glaucoma surgeons considering trabeculectomy surgery in this patient group.

Author contributions GR designed the study, collected the data, analysed the data and wrote the manuscript. JJ involved in study design, collected the data and analysed the data and contributed to reviewing the manuscript. IY analysed the data and contributed to writing the manuscript. JS was involved in study design and writing the manuscript.

\section{Compliance with ethical standards}

Conflict of interest The authors declare that they have no conflict of interest.

\section{References}

1. Tham YC, Li X, Wong TY, Quigley HA, Aung T, Cheng CY. Global prevalence of glaucoma and projections of glaucoma burden through 2040: a systematic review and meta-analysis. Ophthalmology. 2014;121:2081-90.

2. Higginbotham EJ, Gordon MO, Beiser JA, Drake MV, Bennett GR, Wilson MR, et al. The Ocular Hypertension Treatment Study: topical medication delays or prevents primary open-angle glaucoma in African American individuals. Arch Ophthalmol (Chic, Ill: 1960). 2004;122:813-20.

3. De Moraes CG, Demirel S, Gardiner SK, Liebmann JM, Cioffi GA, Ritch R, et al. Effect of treatment on the rate of visual field change in the ocular hypertension treatment study observation group. Invest Ophthalmol Vis Sci. 2012;53:1704-9.

4. Heijl A, Leske MC, Bengtsson B, Hyman L, Bengtsson B, Hussein M. Reduction of intraocular pressure and glaucoma progression: results from the Early Manifest Glaucoma Trial. Arch Ophthalmol (Chic, Ill: 1960). 2002;120:1268-79.

5. Landers J, Martin K, Sarkies N, Bourne R, Watson P. A twenty-year follow-up study of trabeculectomy: risk factors and outcomes. Ophthalmology. 2012;119:694-702.

6. Koseki N, Araie M, Shirato S, Yamamoto S. Effect of trabeculectomy on visual field performance in central 30 degrees field in progressive normal-tension glaucoma. Ophthalmology. 1997;104:197-201.

7. Levene RZ. Central visual field, visual acuity, and sudden visual loss after glaucoma surgery. Ophthalmic Surg. 1992;23: 388-94.

8. Awai M, Koga T, Inatani M, Inoue T, Tanihara H. Stability of the central visual field after modern trabeculectomy techniques in eyes with advanced glaucoma. Jpn $\mathbf{J}$ Ophthalmol. 2007;51:116-20

9. Francis BA, Hong B, Winarko J, Kawji S, Dustin L, Chopra V. Vision loss and recovery after trabeculectomy: risk and associated risk factors. Arch Ophthalmol (Chic, Ill: 1960). 2011;129: 1011-7.

10. Stead R, Azuara-Blanco A, King AJ. Attitudes of consultant ophthalmologists in the UK to initial management of glaucoma patients presenting with severe visual field loss: a national survey. Clin Exp Ophthalmol. 2011;39:858-64.

11. Martinez JA, Brown RH, Lynch MG, Caplan MB. Risk of postoperative visual loss in advanced glaucoma. Am J Ophthalmol. 1993;115:332-7.

12. Law SK, Nguyen AM, Coleman AL, Caprioli J. Severe loss of central vision in patients with advanced glaucoma undergoing trabeculectomy. Arch Ophthalmol (Chic, Ill: 1960). 2007;125: 1044-50. 
13. Markowitz SN, Reyes SV. Microperimetry and clinical practice: an evidence-based review. Can J Ophthalmol J Can d'ophtalmologie. 2013;48:350-7.

14. Lima VC, Prata TS, De Moraes CG, Kim J, Seiple W, Rosen RB, et al. A comparison between microperimetry and standard achromatic perimetry of the central visual field in eyes with glaucomatous paracentral visual-field defects. Br J Ophthalmol. 2010;94:64-7.

15. Chang RT, Knight OJ, Feuer WJ, Budenz DL. Sensitivity and specificity of time-domain versus spectral-domain optical coherence tomography in diagnosing early to moderate glaucoma. Ophthalmology. 2009;116:2294-9.

16. Leung CK, Cheung CY, Weinreb RN, Qiu Q, Liu S, Li H, et al. Retinal nerve fiber layer imaging with spectral-domain optical coherence tomography: a variability and diagnostic performance study. Ophthalmology. 2009;116:1257-63. 63.e1-2

17. Lachenmayr BJ, Drance SM. Central function and visual field damage in glaucoma. Int Ophthalmol. 1992;16:203-9.

18. Schulzer M. Intraocular pressure reduction in normal-tension glaucoma patients. The Normal Tension Glaucoma Study Group. Ophthalmology. 1992;99:1468-70.

19. Comparison of glaucomatous progression between untreated patients with normal-tension glaucoma and patients with therapeutically reduced intraocular pressures. Collaborative NormalTension Glaucoma Study Group. Am J Ophthalmol. 1998;126:487-97.

20. Grunewald F, Bresson-Dumont H, Bechetoille A. Is trabeculectomy without danger in case of threatened fixation?. J Fr d'ophtalmologie. 1996;19:253-8.
21. Costa VP, Smith M, Spaeth GL, Gandham S, Markovitz B. Loss of visual acuity after trabeculectomy. Ophthalmology. 1993;100:599-612.

22. Orzalesi N, Miglior S, Lonati C, Rosetti L. Microperimetry of localized retinal nerve fiber layer defects. Vision Res. 1998;38:763-71.

23. Raghu N, Pandav SS, Kaushik S, Ichhpujani P, Gupta A. Effect of trabeculectomy on RNFL thickness and optic disc parameters using optical coherence tomography. Eye. 2012;26:1131-7.

24. Chang PT, Sekhon N, Budenz DL, Feuer WJ, Park PW, Anderson DR. Effect of lowering intraocular pressure on optical coherence tomography measurement of peripapillary retinal nerve fiber layer thickness. Ophthalmology. 2007;114:2252-8.

25. Maneesang S, Jatutong O, Iemsomboon W. The assessment of retinal nerve fiber layer thickness changing after glaucoma surgery by optical coherence tomography, Phramongkutklao Hospial. J Med Assoc Thailand. 2012;95(Suppl 5):S75-9.

26. Padhy D, Rao A. Change in retinal nerve fiber layer thickness after trabeculectomy. J Glaucoma. 2015;24:252-3.

27. Yamada N, Tomita G, Yamamoto T, Kitazawa Y. Changes in the nerve fiber layer thickness following a reduction of intraocular pressure after trabeculectomy. J Glaucoma. 2000;9:371-5.

28. Lee EJ, Kim TW. Lamina cribrosa reversal after trabeculectomy and the rate of progressive retinal nerve fiber layer thinning. Ophthalmology. 2015;122:2234-42.

29. Higashide T, Ohkubo S, Sugimoto Y, Kiuchi Y, Sugiyama K. Persistent hypotony after trabeculectomy: incidence and associated factors in the Collaborative Bleb-Related Infection Incidence and Treatment Study. Jpn J Ophthalmol. 2016;60:309-18. 